\title{
An update on transcriptional and post-translational regulation of brain voltage-gated sodium channels
}

\author{
Donatus O. Onwuli ${ }^{1} \cdot$ Pedro Beltran-Alvarez ${ }^{1}$
}

Received: 15 October 2015 / Accepted: 16 October 2015 / Published online: 27 October 2015

(C) The Author(s) 2015. This article is published with open access at Springerlink.com

\begin{abstract}
Voltage-gated sodium channels are essential proteins in brain physiology, as they generate the sodium currents that initiate neuronal action potentials. Voltage-gated sodium channels expression, localisation and function are regulated by a range of transcriptional and post-translational mechanisms. Here, we review our understanding of regulation of brain voltage-gated sodium channels, in particular SCN1A $\left(\mathrm{Na}_{\mathrm{V}} 1.1\right), \mathrm{SCN} 2 \mathrm{~A}\left(\mathrm{Na}_{\mathrm{V}} 1.2\right)$, SCN3A $\left(\mathrm{Na}_{\mathrm{V}} 1.3\right)$ and $\mathrm{SCN} 8 \mathrm{~A}\left(\mathrm{Na}_{\mathrm{V}} 1.6\right)$, by transcription factors, by alternative splicing, and by post-translational modifications. Our focus is strongly centred on recent research lines, and newly generated knowledge.
\end{abstract}

Keywords Voltage-gated sodium channel - Regulation · Transcription factor $\cdot$ Alternative splicing $\cdot$ Posttranslational modification

\section{Introduction}

Voltage-gated sodium channels are essential proteins in brain physiology. Upon voltage-mediated activation, sodium channels produce sodium currents responsible for depolarisation of excitable cells, including neurons and cardiomyocytes. From the point of view of biomedical sciences and pathophysiology, brain disorders such as some forms of epilepsy have long been directly associated with voltage-gated sodium channel malfunction.

Pedro Beltran-Alvarez

p.beltran-alvarez@hull.ac.uk

1 School of Biological, Biomedical and Environmental Sciences, University of Hull, Hardy Building Cottingham Road, Hull HU6 7RX, UK
Sodium channels are thought to be macromolecular complexes composed of tens of different proteins (Abriel et al. 2015). The pore-forming protein is known as the $\alpha$ subunit, and is sufficient to generate sodium currents. All $\alpha$ subunits include a voltage sensor that promotes channel opening when the cell membrane is depolarized by a few millivolts. Sodium channels thus activate, generate the sodium currents that underlie the initial depolarisation phase of the action potential, and then inactivate within tens of milliseconds, critically shaping cell repolarisation (Zilberter et al. 1994).

There are nine isoforms of the voltage-gated sodium channel $\alpha$ subunit, and each form has distinct expression and electrophysiological patterns. In this review, we have considered the main sodium channel isoforms expressed in the central neuronal system (CNS), i.e., SCN1A $\left(\mathrm{Na}_{\mathrm{V}} 1.1\right)$, SCN2A ( $\left.\mathrm{Na}_{\mathrm{V}} 1.2\right)$, SCN3A $\left(\mathrm{Na}_{\mathrm{V}} 1.3\right)$ and SCN8A $\left(\mathrm{Na}_{\mathrm{V}} 1.6\right)$. Wherever relevant we have also included additional information regarding other isoforms, including SCN5A (generally known as the cardiac isoform, $\mathrm{Na}_{\mathrm{V}} 1.5$ ) and SCN9A (mainly expressed in the peripheral nervous system, $\mathrm{Na}_{\mathrm{V}}$ 1.7), (Dib-Hajj et al. 2013).

Sodium channel $\alpha$ subunits are large (ca. 2000 residues), hydrophobic, integral membrane proteins that have been fascinating (and challenging) a range of scientific communities including biochemists, pharmacists, neuroscientists, and electrophysiologists for more than three decades (Catterall 2015). Although detailed mammalian voltagegated sodium channel structures are not yet available, it is widely accepted that the topology of $\alpha$ subunits at the protein level consists of four homologous domains (termed DI to DIV), each consisting of six transmembrane helices, and joined by cytosolic interdomain linkers ( $\mathrm{Yu}$ and Catterall 2003). The $N$ and $C$ termini of $\alpha$ subunits are also intracellular. Thus, cytosolic interdomain linkers, and $N$ - and 
$C$-terminal domains of $\alpha$ subunits are accessible to intracellular enzymes that catalyse post-translational modifications (PTM) of the channels.

In this review, we aim to integrate progress in our understanding of CNS voltage-gated sodium channel regulation at the transcriptional and post-translational level. The reader will find that much more is known on sodium channel PTMs than on the transcriptional mechanisms that regulate channel expression. Consequently, the weight of the review is balanced towards PTMs. Our focus is strongly centred on recent research lines, and newly generated knowledge. The goal is to facilitate dissemination of recent developments with a view on fostering further relevant research.

\section{Regulation of brain sodium channel expression at the transcriptional level}

In this section, we have considered the regulation of CNS voltage-gated sodium channels by transcription factors, and by alternative splicing. The regulation of sodium channels at the post-transcriptional level (e.g., by microRNAs) is out of the scope of the present review.

\section{Regulation by transcription factors}

Promoter regions of brain voltage-gated sodium channel genes have been described, including SCN1A (Dong et al. 2014; Long et al. 2008); SCN2A (Lu et al. 1998; Schade and Brown 2000), SCN3A (Martin et al. 2007), and SCN8A (Drews et al. 2005, 2007). Based on the sequence analyses and databases, several transcription factors have been proposed to control brain sodium channel expression (Long et al. 2008). Experimentally, a recent study has shown that $\mathrm{SCN} 3 \mathrm{~A}$ expression is regulated by promoter CpG methylation and Methyl-CpG-binding domain protein 2 (MBD2), ( $\mathrm{Li}$ et al. 2015). MBD2 targets methylated $\mathrm{CpG}$ for demethylation, possibly leading to activated transcription. Consistently, knock-down of MBD2 decreased SCN3A mRNA levels in a neuroblastoma cell line. In seizure-induced mice, MBD2 expression was increased, which correlated with decreased $\mathrm{CpG}$ methylation, and enhanced SCN3A expression (Li et al. 2015).

Another recent development has been the identification of receptor for activated C kinase 1 (RACK1) as a repressor of SCN1A expression (Dong et al. 2014). The authors identified a transcriptional silencer in a region between +53 and +62 bp downstream of SCN1A promotor and used EMSA assays to uncover possible transcriptional regulators. RACK1 was found to bind to the silencer in NT2 cells (a pluripotent embryonal carcinoma cell line often used for differentiation into neurons). Knocking-down RACK1 in
NT2 cells markedly increased SCN1A mRNA levels (Dong et al. 2014).

Sodium channel macromolecular complexes may incorporate proteins classically known as voltage-gated sodium channel $\beta$ subunits. These include five different proteins termed $\beta 1, \beta 1 \mathrm{~b}, \beta 2, \beta 3$, and $\beta 4$. Many groups have studied the effect of $\beta$ subunits on $\alpha$ subunit trafficking and electrophysiology, mainly from the point of view of protein-protein interactions (for a recent review, see Namadurai et al. 2015).

Additionally, sodium channel $\beta$ subunits have been proposed to regulate $\alpha$ subunits at the transcriptional level. One of the first experimental observations was the increase in $\mathrm{Na}_{\mathrm{V}} 1.1 \mathrm{mRNA}$ and protein levels in the presence of proteases targeting the $\beta 2$ subunit. The group of Kovacs, and others, has demonstrated the sequential mechanism by which, first, ADAM10 and BACE1 proteases cleave off the extracellular domain of the $\beta 2$ subunit. Second, $\gamma$-secretase releases the $\beta 2$ intracellular domain. And third, the $\beta 2$ intracellular domain induces an increase in $\mathrm{Na}_{\mathrm{V}} 1.1$ mRNA and protein levels (Kim et al. 2005, 2007; Wong et al. 2005), although the precise pathways for $\beta 2$ internalisation into the cell nucleus remain unknown. BACE1-dependent sodium channel expression seems to be specific for $\mathrm{Na}_{\mathrm{V}} 1.1$, and mRNA levels of other brain $\mathrm{Na}_{\mathrm{V}}$ isoforms including $\mathrm{Na}_{\mathrm{V}} 1.2, \mathrm{Na}_{\mathrm{V}} 1.3$ and $\mathrm{Na}_{\mathrm{V}} 1.6$ are relatively insensitive to BACE1 protease activity (Kim et al. 2007, 2011).

Likewise $\beta 2$, the $\beta 1$ subunit has been shown to regulate $\mathrm{Na}_{\mathrm{V}}$ expression, and mouse models show changes in brain $\mathrm{Na}_{\mathrm{V}}$ expression and localization upon $\beta 1$ deletion (Chen et al. 2004). In a recent development, $\beta 1$ subunit silencing has been shown to result in decreased $\mathrm{Na}_{\mathrm{V}} 1.1, \mathrm{Na}_{\mathrm{V}} 1.3$ and $\mathrm{Na}_{\mathrm{V}} 1.6$ (but not $\mathrm{Na}_{\mathrm{V}} 1.2$ ) mRNA and protein levels in cells models (Baroni et al. 2014), although the mechanism underlying this regulation was not investigated. Although $\beta 1$ subunit is a target for BACE1 in vitro, the question remains whether this is physiologically relevant (Wong et al. 2005).

\section{Regulation by alternative splicing}

The first evidences for alternative splicing of brain sodium channels were reported more than 20 years ago (Sarao et al. 1991; Gustafson et al. 1993), and splicing mechanisms are thought to be common to most brain $\mathrm{Na}_{\mathrm{V}}$ isoforms (Copley 2004). In particular, SCN1A alternative splicing has been extensively studied due to its relevance in CNS disorders such as epilepsy (Lossin 2009; Schlachter et al. 2009; Le Gal et al. 2011; Thompson et al. 2011).

The best studied SCN1A splicing variants are often referred to as the adult and neonatal forms, although both forms are expressed in adults. They result from the mutually exclusive expression of either exon $5 \mathrm{~A}$ (adult) or $5 \mathrm{~N}$ 
(neonatal). Common SCN1A polymorphisms can have a massive effect on the expression of the $5 \mathrm{~N}$ variant in normal adults (Tate et al. 2005; Heinzen et al. 2007). The $5 \mathrm{~A} / 5 \mathrm{~N}$ splicing event can also be modulated by splicemodifier proteins, including sodium channel modifier 1 (SCNM1). Very recently, a mutation in SCNM1 has been linked to epilepsies possibly via regulation of SCN1A splicing leading to reduction of the variant containing exon 5N (Kasteleijn-Nolst Trenité et al. 2015). SCNM1, as well as other splicing regulators such as Rbfox2, can also modulate SCN8A splicing (Buchner et al. 2003; Gehman et al. 2012)

\section{Regulation of brain sodium channels at the post-translational level}

From biochemical assays in vitro to targeted purification of proteins from tissues, research in sodium channel PTMs has recently expanded from (immuno) chemical methods to embrace mass spectrometry and proteomics. Here, we review our current understanding of some of the best known sodium channel PTMs. As before, we have included $\mathrm{Na}_{\mathrm{V}}$ 1.1, $\mathrm{Na}_{\mathrm{V}} 1.2, \mathrm{Na}_{\mathrm{V}} 1.3$, and $\mathrm{Na}_{\mathrm{V}}$ 1.6. Where relevant, $\mathrm{Na}_{\mathrm{V}} 1.5$ has also been considered because of the wealth of available $\mathrm{Na}_{\mathrm{V}} 1.5$ PTM data. In particular, $\mathrm{Na}_{\mathrm{V}} 1.5$ phosphorylation, ubiquitylation, and arginine methylation have been studied in detail ("Phosphorylation", "Ubiquitylation" and "Arginine methylation", respectively).

Previously in "Regulation by transcription factors", we have reviewed our knowledge of sodium channel $\beta$ subunit processing by proteases, leading to transcriptional regulation of $\alpha$ subunits. In "Regulation of brain sodium channels by proteases", we have included available data on direct proteolysis of $\alpha$ subunits. Although proteolysis is in most cases associated with degradation, it can also be regarded as PTM if it is limited and specific (Rogers and Overall 2013).

\section{Phosphorylation}

Phosphorylation is the most experimentally observed PTM at the proteome-wide level, and it is certainly thought to be the most abundant PTM along with $N$-glycosylation (Khoury et al. 2011). Sodium channels are no exception to the rule and phosphorylation is the most studied and observed sodium channel PTM.

\section{Identified phosphorylation sites}

The aim of this subsection is to comprehensively collect and update the repertoire of sodium channel 'phosphorylatable' sites (Table 1). These include phosphosites identified by the use of in vitro assays and heterologous expression experiments, as well as those identified in sodium channels isolated from native sources. Data in Table 1 are taken from classical papers (Berendt et al. 2010), and previous reviews (Cerda et al. 2011; Baek et al. 2011), and updated to include recent original articles that described novel phosphosites (Marionneau et al. 2012; Baek et al. 2014; Herren et al. 2015). Functional consequences of $\mathrm{Na}_{\mathrm{V}}$ phosphorylation are discussed below.

Is it safe to assume that if a $\mathrm{Na}_{\mathrm{V}}$ isoform is phosphorylated at a certain residue, then our favourite isoform will also be, provided the site is conserved? The general answer is No. Visual analysis of phosphosite conservation in Table 1 leaves little room for hope, at least according to the data currently available. The exception is the interdomain linker between domains I and II, which is considered the PTM hot-spot (Cantrell and Catterall 2001), and where one can find phosphosites conserved among 3, sometimes 4 , of the considered $\mathrm{Na}_{\mathrm{V}}$ isoforms. Nevertheless, due to the substoichiometric and labile nature of phosphorylation, the failure to detect a protein modification does not imply that a residue is not phosphorylated. Perhaps future comprehensive proteomic studies will demonstrate higher degree in phosphosite conservation among $\mathrm{Na}_{\mathrm{V}}$ isoforms.

\section{Specificity of the functional effect of phosphorylation among $\mathrm{Na}_{V}$ isoforms}

Most of the phosphosites included in Table 1 were identified by proteomics and mass spectrometry methods, and we currently lack information on which protein kinase may catalyse phosphorylation of many of the $\mathrm{Na}_{\mathrm{V}}$ phosphosites. Nevertheless, it has long been known that protein kinase $\mathrm{C}$ (PKC) and cAMP-dependent kinase (PKA) can phosphorylate brain $\mathrm{Na}_{\mathrm{V}}$ channels (West et al. 1991; Numann et al. 1991; Li et al. 1992, 1993). Other kinases involved in regulating brain $\mathrm{Na}_{\mathrm{V}}$ phosphorylation are glycogen synthase kinase 3 (GSK3) (James et al. 2015), protein kinase CK2 (Hien et al. 2014), A kinase-anchoring protein 15 (Few et al. 2007), Fyn tyrosine kinase (Beacham et al. 2007), and p38 mitogen-kinase activated protein kinase (Wittmack et al. 2005).

The functional effects of channel phosphorylation on $\mathrm{Na}_{\mathrm{V}}$ electrophysiology often depend on the specific isoform of interest. For instance, phosphorylation by PKA and PKC results in attenuation of $\mathrm{Na}_{\mathrm{V}} 1.2$ currents due to defective channel trafficking to the cell surface (Li et al. 1992). But $\mathrm{Na}_{\mathrm{V}} 1.6$ channels are relatively insensitive to PKA/ PKC regulation (Chen et al. 2008), and $\mathrm{Na}_{\mathrm{V}} 1.5$ currents are enhanced by PKA activation due to increased $\mathrm{Na}_{\mathrm{V}} 1.5$ expression at the cell surface (Hallaq et al. 2006). Subtle variations in the primary sequence of $\mathrm{Na}_{\mathrm{V}}$ isoforms must underlie such differences. For instance, it is thought that 
Table 1 Phosphosites of different $\mathrm{Na}_{\mathrm{V}}$ isoforms

MEQTVLVPPG PDSENFFTRE SLAAIERRIA EEKAKNPKPD ----KKDDDE MARSVLVPPG PDSFRFFTRE SLAAIEQRIA EEKAKRPKQE ---RKDEDDE MAQALLVPPG PESFRLFTRE SLAAIEKRAA EEKAKKPKKE ----QDNDDE M-ANFLLPRG TSSFRRFTRE SLAAIEKRMA EKQARGSTTL QESREGLPEE MAARVLAPPG PDSFKPFTPE SLANIERRIA ESKLKKPPKA DGSHREDDED

NGPKPNSDLE AGKNLPFIYG DIPPEMVSEP LEDLDPYYIN KKTFIVLNKG NGPKPNSDLE AGKSLPFIYG DIPPEMVSEP LEDLDPYYIN KKTFIVLNKG NKPKPNSDLE AGKNLPFIYG DIPPEMVSEP LEDLDPYYIN KKTFIVMNKG EAPRPQLDLQ ASKKLPDLYG NPPQELIGEP LEDLDPFYST QKTFIVLNKG SKPKPNSDLE AGKSLPFIYG DIPQGLVAVP LEDFDPYYLT QKTFVVLNRG

KAIFRFSATS ALYILTPFNP LRKIAIKILV HSLFSMLIMC TILTNCVFMT KAISRFSATS ALYILTPFNP IRKLAIKILV HSLFNVLIMC TILTNCVFMT KAIFRFSATS ALYILTPLNP VRKIAIKILV HSLFSMLIMC TILTNCVFMT KTIFRFSATN ALYVLSPFHP IRRAAVKILV HSLFNMLIMC TILTNCVFMA KTLFRFSATP ALYILSPFNL IRRIAIKILI HSVFSMIIMC TILTNCVFMT

EFOQMLEQLK KQOEAAOQAA ATTASE---- -HSRE---PS AAGRLSDSSS EFQQMLEQLK KQQEEAQ-AA AAAASA---- -ESRDFSGAG GIGVFSESSS EFQQMLEQLK KQQEEAQ--A VAAASA---- -ASRDFSGIG GLGELLESSS RFQEAMEMLK KEHEALT--- --------- -------IR GVDTVSRSSL EFKAMLEQLK KQQEEAQ-AA AMATSAGTVS EDAIEEEGED GVGS-PRSSS

EASKLSSKSA KERRNRRKKR KQKEQSGGEE K-DDDEFHKS ESEDSIRRKG VASKLSSKSE KELKNRRKKK KOKEQAGEEE K--EDAVRKS ASEDSIRKKG EASKLSSKSA KEWRNRRKKR RQREHLEGNN KGERDSFPKS ESEDSVKRSS EMSPLAPVNS HERRSKRRKR ----MSSGTE ECGEDRLPKS DSEDGPR--ELSKLSSKSA KERRNRRKKR KOKELSEGEE KGDPEKVFKS ESEDGMRRKA

FRFSIEGNRL TYEKRYSSPH QSLLSIRGSL FSPRRNSRTS LFSFR--GRA FQFSLEGSRL TYEKRFSSPH QSLLSIRGSL FSPRRNSRAS LFNFK--GRV FLFSMDGNRL TSDKKFCSPH OSLLSIRGSL FSPRRNSKTS IFSFR--GRA ------- -----AMNH LSLTRGLSRT SMKPRSSRGS IFTFR--R-FR--LPDNRI G--RKFSIMN QSLLSIPGSP FLSRHNSKSS IFSFRGPGRF

KDVGSENDFA DDEHSTFEDN ESRRDSLFVP RRHGERRN-- ---SNLSQTS KDIGSENDFA DDEHSTFEDN DSRRDSLFVP HRHGERRP-- ---SNVSOAS KDVGSENDFA DDEHSTFEDS ESRRDSLFVP HRHGERRN-- ---SNVSQAS RDLGSEADFA DDENSTAGES ESHHTSLLVP --WPLRRT-- ---SAQGQPS RDPGSENEFA DDEHSTVEES EGRRDSLFIP IRARERRSSY SGYSGYSQCS

RSSRMLAVFP ANGKMHSTVD CNGVVSLVG- GPSVPTSPVG QLLPEVIIDK RASRGIPTLP MNGKMHSAVD CNGVVSLVG- GPSALTSPVG QLLPE----MSSRMVPGLP ANGKMHSTVD CNGVVSLVG- GPSALTSPTG QLPPE----PGTSA-PGHA LHGKKNSTVD CNGVVSLLGA GDPEATSPGS HLLRPVMLEH RSSRIFPSLR RSVKRNSTVD CNGVVSLIG- ----PGSHIG RLLPE-----

PATDDNGTTT ETEMRKRRSS SFHVSMDFLE DPSQRQRAMS IASILTNTV-----GTTT ETEIRKRRSS SYHVSMDLLE DP-SRQRAMS MASILTNTM------GTTT ETEVRKRRLS SYQISMEMLE DSSGRQRAVS IASILTNTMPP--DTTTPS EEPGGPQMLT SQAPCVDGFE EPGARQRALS AVSVLTSAL------ATT EVEIKKKGPG SLLVSMEQLA SYGRKDRINS IMSVVTNTLV

EELEESROKC PPCWYKFSNI FLIWDCSPYW LKVKHIVNLV VMDPFVDLAI EELEESRQKC PPCWYKFANM CLIWDCCKPW LKVKHVVNLV VMDPFVDLAI EELEESRQKC PPCWYRFANV FLIWDCCDAW LKVKHLVNLI VMDPFVDLAI EELEESRHKC PPCWNRLAQR YLIWECCPLW MSIKQGVKLV VMDPFTDLTI EELEESORKC PPCWYKFANT FLIWECHPYW IKLKEIVNLI VMDPFVDLAI
$46 \mathrm{Na}_{\mathrm{v}} 1.1$ mouse (N terminus) $47 \mathrm{Na}_{\mathrm{v}} 1.2$ rat (N terminus) $46 \mathrm{Na}_{\mathrm{v}} 1.3$ human (N terminus) $49 \mathrm{Nav}_{\mathrm{v}} 1.5$ human (N terminus) $50 \mathrm{Na}_{\mathrm{v}} 1.6$ mouse (N terminus)

$96 \mathrm{Na}_{\mathrm{v}} 1.1$ mouse (N terminus) $97 \mathrm{Na}_{\mathrm{v}} 1.2$ rat (N terminus) $96 \mathrm{Na}_{\mathrm{v}} 1.3$ human (N terminus) $99 \mathrm{Na}_{\mathrm{v}} 1.5$ human (N terminus) $100 \mathrm{Na}_{\mathrm{v}} 1.6$ mouse (N terminus)

$146 \mathrm{Na}_{\mathrm{v}} 1.1$ mouse (N terminus) $147 \mathrm{Na}_{\mathrm{v}} 1.2$ rat (N terminus) $146 \mathrm{Na}_{\mathrm{v}} 1.3$ human (N terminus) $149 \mathrm{Na}_{\mathrm{v}} 1.5$ human (N terminus) $150 \mathrm{Na}_{\mathrm{v}} 1.6$ mouse (N terminus)

484 Nav1.1 mouse (Linker DI-DII) $488 \mathrm{Na}_{\mathrm{v}} 1.2$ rat (Linker DI-DII) $486 \mathrm{Na}_{\mathrm{v}} 1.3$ human (Linker DI-DII) $461 \mathrm{Na}_{\mathrm{V}} 1.5$ human (Linker DI-DII) $478 \mathrm{Na}_{\mathrm{v}} 1.6$ mouse (Linker DI-DII)

533 NaV1.1 mouse (Linker DI-DII) 536 NaV1.2 rat (Linker DI-DII) 536 NaV1.3 human (Linker DI-DII) 504 NaV1.5 human (Linker DI-DII) 528 NaV1.6 mouse (Linker DI-DII)

$581 \mathrm{Na}_{\mathrm{v}} 1.1$ mouse (Linker DI-DII) $584 \mathrm{Na}_{\mathrm{V}} 1.2$ rat (Linker DI-DII) $584 \mathrm{Na}_{\mathrm{v}} 1.3$ human (Linker DI-DII) $534 \mathrm{Na}_{\mathrm{V}} 1.5$ human (Linker DI-DII) 574 Nav1.6 mouse (Linker DI-DII)

$626 \mathrm{Na}_{\mathrm{v}} 1.1$ mouse (Linker DI-DII) $629 \mathrm{Na}_{\mathrm{v}} 1.2$ rat (Linker DI-DII) $629 \mathrm{Na}_{\mathrm{V}} 1.3$ human (Linker DI-DII) 577 Nav1.5 human (Linker DI-DII) $624 \mathrm{Na}_{\mathrm{v}} 1.6$ mouse (Linker DI-DII)

$675 \mathrm{Na}_{\mathrm{V}} 1.1$ mouse (Linker DI-DII) $673 \mathrm{Na}_{\mathrm{V}} 1.2$ rat (Linker DI-DII) $673 \mathrm{Na}_{\mathrm{V}} 1.3$ human (Linker DI-DII) $626 \mathrm{Na}_{\mathrm{v}} 1.5$ human (Linker DI-DII) $664 \mathrm{Na}_{\mathrm{v}} 1.6$ mouse (Linker DI-DII)

724 Nav1.1 mouse (Linker DI-DII) $715 \mathrm{Na}_{\mathrm{v}} 1.2$ rat (Linker DI-DII) $716 \mathrm{Na}_{\mathrm{V}} 1.3$ human (Linker DI-DII) 673 Nav1.5 human (Linker DI-DII) 707 Nav1.6 mouse (Linker DI-DII)

$774 \mathrm{Na}_{\mathrm{v}} 1.1$ mouse (Linker DI-DII) 765 Nav1.2 rat (Linker DI-DII) $766 \mathrm{Na}_{\mathrm{V}} 1.3$ human (Linker DI-DII) $723 \mathrm{Na}_{\mathrm{V}} 1.5$ human (Linker DI-DII) 757 Nav1.6 mouse (Linker DI-DII) 
Table 1 continued

YVKRKIYEFI QQSFVKKQKI LDEIKPLDDL NNRKDNCISN HT----TEIG FVKRKIREFI QKAFVRKQKA LDEIKPLEDL NNKKDSCISN HTT---IEIG YVKNKMRECF QKAFFRKPKV IEIHE----- GNKIDSCMSN NTG---IEIS FVKRTTWDFC CGLLRQRPQK PAALAAQGQL ----PSCIAT PYSPPPPETE WAKVKVHAFM QAHF--KQRE ADEVKPLDEL YEKKANCIAN HTG---VDIH

KDLDCLKDV- --NGTTSGIG TGSSVEKYII DESDYMSFIN NPSLTVTVPI KDLNYLKDG- - -NGTTSGI- -GSSVEKYVV DESDYMSEIN NPSLTVTVPI KELNYLRDG- --NGTTSGVG TGSSVEKYVI DENDYMSEIN NPSLTVTVPI KVPPTRKETR FEEGEQPGQG TPGDPE---- -------- ----PVCVPI RNGDFQKNG- --NGTTSGI- -GSSVEKYII DE-DHMSFIN NPNLTVRVPI

AVGESDFENL NTEDFSSESD LEESKEKLNE ALGESDFENL NTEEFSSESD MEESKEKLNAVGESDFENL NTEEFSSESE LEESKEKLNAVAESDTDDQ EEDEENSLGT EEESSKQQES QPVSGGPEAP PDSRTWSQVS AVGESDFENL NTEDVSSESD PEGSKDKLD-
$1070 \mathrm{Na}_{\mathrm{v}} 1.1$ mouse (Linker DII-DIII) $1062 \mathrm{Na}_{\mathrm{v}} 1.2$ rat (Linker DII-DIII) $1058 \mathrm{Na}_{\mathrm{V}} 1.3$ human (Linker DII-DIII) $1017 \mathrm{Na}_{\mathrm{v}} 1.5$ human (Linker DII-DIII) $1052 \mathrm{Na}_{\mathrm{V}} 1.6$ mouse (Linker DII-DIII)
LYFVIFIIFG SFFTLNLFIG VIIDNFNQQK KKFGGQDIFM TEEQKKYYNA LYFVIFIIFG SFFTLNLFIG VIIDNFNOQK KKFGGQDIFM TEEQKKYYNA LYFVIFIIFG SFFTLNLFIG VIIDNFNQQK KKFGGQDIFM TEEQKKYYNA IYFVIFIIFG SFFTLNLFIG VIIDNFNOQK KKLGGODIFM TEEOKKYYNA IYFVIFIIFG SFFTLNLFIG VIIDNFNQQK KKFGGQDIFM TEEQKKYYNA
$1117 \mathrm{Na}_{\mathrm{v}} 1.1$ mouse (Linker DII-DIII) $1107 \mathrm{Na}_{\mathrm{v}} \mathrm{l} .2$ rat (Linker DII-DIII) $1105 \mathrm{Na}_{\mathrm{v}} 1.3$ human (Linker DII-DIII) 1049 Nav1.5 human (Linker DII-DIII) $1096 \mathrm{Na}_{\mathrm{v}} 1.6$ mouse (Linker DII-DIII)
MKKLGSKKPQ KPIPRPGNKF QGMVFDFVTR QVFDISIMIL ICLNMVTMMV MKKLGSKKPQ KPIPRPANKF QGMVFDFVTK QVFDISIMIL ICLNMVTMMV MKKLGSKKPQ KPIPRPANKF QGMVFDFVTR QVFDISIMIL ICLNMVTMMV MKKLGSKKPQ KPIPRPLNKY OGFIFDIVTK OAFDVTIMFL ICLNMVTMMV MKKLGSKKPQ KPIPRPLNKI QGIVFDFVTQ QAFDIVIMML ICLNMVTMMV

\section{Nav1.1 mouse (Linker DII-DIII) $1136 \mathrm{Na}_{\mathrm{v}} 1.2$ rat (Linker DII-DIII) $1134 \mathrm{Na}_{\mathrm{v}} 1.3$ human (Linker DII-DIII) 1099 Nav1.5 human (Linker DII-DIII) $1125 \mathrm{Na}_{\mathrm{V}} 1.6$ mouse (Linker DII-DIII)}

\begin{abstract}
RIHCLDILFA FTKRVLGESG EMDALRIQME ERFMASNPSK VSYQPITTTL RIHCLDILFA FTKRVLGESG EMDALRIQME ERFMASNPSK VSYEPITTTL RIHCLDILFA FTKRVLGESG EMDALRIQME DRFMASNPSK VSYEPITTTL RIHCMDILFA FTKRVLGESG EMDALKIQME EKFMAANPSK ISYEPITTTL RIHCLDILFA FTKRVLGDSG ELDILRQQME ERFVASNPSK VSYEPITTTL

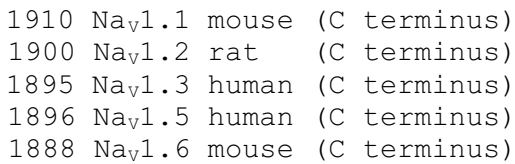

KRKQEEVSAV IIQRAYRRHL LKRTVKQASF TYNKNKL-KG --GANLLVKE KRKQEEVSAI VIQRAYRRYL LKQKVKKVSS IYKKDKG-KE --DEGTPIKE KRKQEEVSAA IIQRNFRCYL LKQRLKNISS NYNKEAI-KG --RIDLPIKQ RRKHEEVSAM VIORAFRRHL LORSLKHASF LFROOAG-SG LSEEDAPERE RRKQEEVSAV VLQRAYRGHL ARRGF----- --------- ------ICR

DMLIDRI-NE N----SITEK TDLTMSTAAC PPSYDRVTKP IVEKHE---Q DIITDKL-NE N----STPEK TDVTPSTTS- PPSYDSVTKP EKEKFE---K DMIIDKL-NG N----STPEK TDGSSSTTS- PPSYDSVTKP DKEKFE---K GLIAYVM-SE NFSRPLGPPS SSSISSTSF- PPSYDSVTRA TSDNLQVRGS KITSNKLENG G----THREK KESTPSTAS- LPSYDSVTKP DKEKQQRAEE
$1957 \mathrm{Na}_{\mathrm{v}} 1.1$ mouse (C terminus) $1947 \mathrm{Na}_{\mathrm{v}} 1.2$ rat (C terminus) $1942 \mathrm{Na}_{\mathrm{v}} 1.3$ human (C terminus) $1945 \mathrm{Na}_{\mathrm{v}} 1.5$ human (C terminus) 1916 Nav1.6 mouse (C terminus)
$1999 \mathrm{Na}_{\mathrm{v}} 1.1$ mouse (C terminus) $1988 \mathrm{Na}_{\mathrm{v}} 1.2$ rat (C terminus) $1983 \mathrm{Nav}_{\mathrm{v}} 1.3$ human (C terminus) $1993 \mathrm{Na}_{\mathrm{v}} 1.5$ human (C terminus) $1961 \mathrm{Na}_{\mathrm{v}} 1.6$ mouse (C terminus)
1510 Nav1.1 mouse (Linker DIII-DIV) $1500 \mathrm{Na}_{\mathrm{v}} 1.2$ rat (Linker DIII-DIV) $1495 \mathrm{Na}_{\mathrm{V}} 1.3$ human (Linker DIII-DIV) $1497 \mathrm{Na}_{\mathrm{v}} 1.5$ human (Linker DIII-DIV) $1489 \mathrm{Na}_{\mathrm{V}} 1.6$ mouse (Linker DIII-DIV)
$1560 \mathrm{Na}_{\mathrm{V}} 1.1$ mouse (Linker DIII-DIV) $1550 \mathrm{Na}_{\mathrm{V}} 1.2$ rat (Linker DIII-DIV) $1545 \mathrm{Na}_{\mathrm{V}} 1.3$ human (Linker DIII-DIV) $1547 \mathrm{Na}_{\mathrm{V}} 1.5$ human (Linker DIII-DIV) $1539 \mathrm{Na}_{\mathrm{V}} 1.6$ mouse (Linker DIII-DIV)

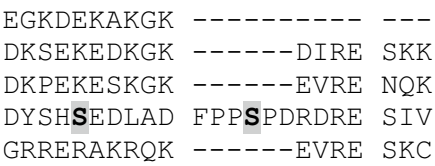

Phosphorylated residues are shown bold and shadowed
$\mathrm{Na}_{\mathrm{V}} 1.5$ phosphorylation by PKA at S528 masks an endoplasmic reticulum retention signal $\left(\mathrm{RRR}_{535}\right)$, thereby promoting $\mathrm{Na}_{\mathrm{V}} 1.5$ trafficking to the membrane (Zhou et al. 2002). This endoplasmic reticulum signal is absent in $\mathrm{Na}_{\mathrm{V}} 1.2\left(\mathrm{RVK}_{585}\right)$ and modified in $\mathrm{Na}_{\mathrm{V}} 1.6\left(\mathrm{RFR}_{575}\right)$.
Opposite functional effects of post-translational modifications on distinct $\mathrm{Na}_{\mathrm{V}}$ isoforms have also been observed after phosphorylation by Fyn kinase. Fyn kinase phosphorylates essential tyrosine residues within the inactivation gate of sodium channels, including the equivalent Y1498 
$\left(\mathrm{Na}_{\mathrm{V}} 1.2\right)$ and $\mathrm{Y} 1495\left(\mathrm{Na}_{\mathrm{V}} 1.5\right)$. Yet, the functional effect of phosphorylation by Fyn on channel inactivation is a negative $\left(\mathrm{Na}_{\mathrm{V}} 1.2\right)$ or positive $\left(\mathrm{Na}_{\mathrm{V}} 1.5\right)$ shift in the voltage dependence of inactivation (Beacham et al. 2007; Ahern et al. 2005). The simplest explanation is that Fyn phosphorylates other Tyr residues within $\mathrm{Na}_{\mathrm{V}} 1.2$ and $\mathrm{Na}_{\mathrm{V}} 1.5$ sequences, and this has indeed been demonstrated for $\mathrm{Na}_{\mathrm{V}} 1.2$ (including Y66, Y1497, and Y1893), (Beacham et al. 2007). Nevertheless, recent work has reported that distinct splicing variants of the same $\mathrm{Na}_{\mathrm{V}}$ isoform show different electrophysiological behaviour upon phosphorylation by Fyn, which introduces another level of complexity (Iqbal et al. 2015).

\section{Ubiquitylation}

Protein ubiquitylation (or ubiquitination) is a post-translational modification that involves the orchestrated function of three types of enzymes. First, ubiquitin activating enzyme (E1) catalyses thioester formation between the $C$ terminus of ubiquitin and an internal cysteine. Second, activated ubiquitin is transferred to the ubiquitin conjugating enzyme (E2). Third, ubiquitylation of the substrate protein is catalysed by ubiquitin ligases (E3), which covalently attach ubiquitin molecules to lysine residues within the target sequence. Ubiquitylation is often associated with protein degradation.

There are hundreds of $\mathrm{E} 3$ ubiquitin ligases, usually classified into two groups: HECT (homologous to E6-AP C terminus) ligases, and RING (really interesting new gene) ligases (Goel et al. 2015). Until 2015, it was thought that only HECT ligases could catalyse sodium channel ubiquitylation (see below).

The most studied molecular mechanism for sodium channel ubiquitylation involves channel recognition by Nedd4-2 ubiquitin ligases (HECT-type ligases) via proteinprotein interaction between the WW4 domain of Nedd4-2, and the PY motif of neuronal and cardiac sodium channels (Fotia et al. 2004; van Bemmelen et al. 2004). Ubiquitylation by Nedd4-2 has been shown to tag sodium channels for internalisation from the cell surface, including $\mathrm{Na}_{\mathrm{V}} 1.2$ (Fotia et al. 2004), $\mathrm{Na}_{\mathrm{V}} 1.6$ (Gasser et al. 2010), and $\mathrm{Na}_{\mathrm{V}} 1.5$ (Rougier et al. 2005). However, in most cases, the precise modification site(s), i.e., the Lys residues that are ubiquitylated, remain to be confirmed.

Very recently, compelling evidence has been presented that shows ubiquitylation of sodium channels in zebra fish CNS by RNF121, a member of the RING family of E3 ubiquitin ligases (Ogino et al. 2015). From the initial observation that zebra fish bearing mutations in RNF121 present defective $\mathrm{Na}_{\mathrm{V}}$ trafficking in neurons and skeletal muscle, the investigators moved on to perform heterologous expression of $\mathrm{Na}_{\mathrm{V}} 1.6$ and RNF121 in HEK 293T cells. Results showed increased $\mathrm{Na}_{\mathrm{V}} 1.6$ degradation upon co-expression of RNF121 but, intriguingly, enhanced $\mathrm{Na}_{\mathrm{V}} 1.6$ membrane localization when co-expressed with RNF121 and auxiliary $\mathrm{NaV} \beta$ subunits (Ogino et al. 2015).

\section{Arginine methylation}

Arginine methylation consists on the addition of methyl groups to arginine residues of proteins. Arginine methylation is catalysed by protein arginine methyl transferases (PRMTs) that transfer a methyl group from $S$-adenosyl$L$-methionine (SAM) to the target arginine. Arginine methylation has recently been reported as a novel post-translational modification of the voltage-gated sodium channel family using $\mathrm{Na}_{\mathrm{V}} 1.5$ as a model system (Beltran-Alvarez et al. 2011).

The groups of Comb and Trimmer have described arginine methylation of brain sodium channels. Using a proteomic approach and bespoke antibodies that recognise peptides bearing methylated arginine, the group of Comb reported arginine methylation of $\mathrm{Na}_{\mathrm{V}} 1.1, \mathrm{Na}_{\mathrm{V}} 1.2$ and $\mathrm{Na}_{\mathrm{V}} 1.5$ in the mouse brain (Guo et al. 2014). In parallel, the group of Trimmer described arginine methylation of $\mathrm{Na}_{\mathrm{V}} 1.2$ purified from rat brain (Baek et al. 2014). We analysed the methylation sites reported by the three referenced articles (Beltran-Alvarez et al. 2011; Guo et al. 2014; Baek et al. 2014), and found that three sites have been observed by at least two independent studies (Table 2).

The functional consequences of sodium channel modification by arginine methylation have been documented. Available electrophysiological data are consistent with an increase in sodium current density, most likely due to enhanced $\mathrm{Na}_{\mathrm{V}}$ membrane expression (Beltran-Alvarez et al. 2013; Baek et al. 2014). Additionally, the group of Trimmer reported considerable acceleration in $\mathrm{Na}_{\mathrm{V}} 1.2$ recovery from inactivation when arginine methylation was enhanced (Baek et al. 2014). Remarkably, arginine methylation is an example of PTM conservation among $\mathrm{Na}_{\mathrm{V}}$ isoforms, even if catalysed by different enzymes: $\mathrm{Na}_{\mathrm{V}} 1.2$ is methylated by PRMT8 (mostly expressed in the CNS), while $\mathrm{Na}_{\mathrm{V}} 1.5$ methylation is catalysed by PRMT3 and -5 (ubiquitously expressed).

\section{Other known post-translational modifications}

We would like to mention that sodium channels have long been known to undergo cysteine modifications including $S$-palmitoylation (Schmidt and Catterall 1987; Bosmans et al. 2011), and S-nitrosylation (Renganathan et al. 2002). Methionine oxidation of sodium channels has previously been reviewed (Cui et al. 2012). SUMOylation of the $\mathrm{Na}_{\mathrm{V}} 1.7$ isoform has been described, but available data suggest that SUMOylation may not be conserved in $\mathrm{CNS} \mathrm{Na}_{\mathrm{V}}$ isoforms (Dustrude et al. 2013). 
Another well-known PTM, $N$-glycosylation, has been mostly studied in the cardiac isoform of the sodium channel, and several excellent reviews have recently been published (Baycin-Hizal et al. 2014; Marionneau and Abriel 2015). Perhaps the latest studies are those from the Chatelier and the Decosterd-Abriel groups, which have proposed alternative trafficking pathways for differentially glycosylated $\mathrm{Na}_{\mathrm{V}}$, using $\mathrm{Na}_{\mathrm{V}} 1.5$ and $\mathrm{Na}_{\mathrm{V}} 1.7$ as study models (Mercier et al. 2015; Laedermann et al. 2013, respectively).

\section{Other possible post-translational modifications?}

The advent of large-scale proteomics including the publication of human proteome maps is revolutionising life sciences. The ion channel field can also benefit from the analysis of big data to anticipate and identify challenges and opportunities, particularly in the field of PTMs. With this in mind, we searched Phosphositeplus (Hornbeck et al. 2015) for PTMs of $\mathrm{Na}_{\mathrm{V}}$ isoforms. The database contains potentially novel sodium channel modifications including Lys acetylation, which is reported for $\mathrm{Na}_{\mathrm{V}} 1.1, \mathrm{Na}_{\mathrm{V}} 1.2$, $\mathrm{Na}_{\mathrm{V}} 1.3, \mathrm{Na}_{\mathrm{V}} 1.5$ and $\mathrm{Na}_{\mathrm{V}} 1.6$, and Lys methylation, which is included for $\mathrm{Na}_{\mathrm{V}} 1.2$ and $\mathrm{Na}_{\mathrm{V}} 1.6$.

Although promising at first sight, available data must be regarded with care. Conservation of the reported post-translationally acetylated or methylated Lys site among $\mathrm{Na}_{\mathrm{V}}$ isoforms was very low. The finding worth mentioning was interspecies conservation of $\mathrm{Na}_{\mathrm{V}} 1.1$ acetylation at K1948 in human and mouse samples. Although K1948 acetylation was observed in unrelated experiments, it must be noted that the source of tissue was not brain but colon cancer.

\section{Cross-talk between sodium channel PTMs}

Cross-talk, or interplay, between PTMs includes the regulatory mechanisms by which PTMs work together to determine protein function. Cross-talk between sodium channel phosphorylation, and arginine methylation, has been reported. The group of Trimmer reported cross-talk between $\mathrm{Na}_{\mathrm{V}} 1.2$ arginine methylation and phosphorylation (Baek et al. 2014). In this study, the authors studied $\mathrm{Na}_{\mathrm{V}} 1.2$ PTMs in the rat brain. $\mathrm{Na}_{\mathrm{V}} 1.2$ was immunopurified, digested and subjected to mass spectrometry analysis. An initial observation was that detected $\mathrm{Na}_{\mathrm{V}} 1.2$ peptides harboured either arginine methylation or phosphorylation, but not both PTMs on the same peptide. Convincingly, these two PTMs were reciprocally regulated in response to acute seizure: e.g., R563 methylation (see also Table 2) increased but S554 and S568 phosphorylation decreased after induction of seizure in rats (Baek et al. 2014). The most likely mechanism for this interplay between sodium channel arginine methylation and phosphorylation is the modification of kinetic specificity constants of serine phosphorylation 
upon methylation of a neighbouring arginine, and viceversa (Beltran-Alvarez et al. 2015). Nevertheless, the functional consequences of phosphorylation-arginine methylation cross-talk remain to be elucidated.

Additionally, cross-regulation between $\mathrm{Na}_{\mathrm{V}} 1.6$ phosphorylation, and ubiquitylation, has been observed. On the one hand, $\mathrm{Na}_{\mathrm{V}} 1.6$ is phosphorylated by $\mathrm{p} 38$ MAPK at position $\mathrm{S} 553$. On the other, $\mathrm{Na}_{\mathrm{V}} 1.6$ is ubiquitylated by Nedd4-2 after recognition of the PY motif (Pro-Ser-Tyr) at the $C$ terminus of the channel. Results from the group of Dib-Hajj suggested that S553 phosphorylation enables further $\mathrm{Na}_{\mathrm{V}} 1.6$ ubiquitylation and internalisation of the channel (Gasser et al. 2010). A similar mechanism has recently been proposed for $\mathrm{Na}_{\mathrm{V}} 1.2$ whereby phosphorylation of T1966 by GSK3 primes recognition by Nedd4-2 via the $\mathrm{Na}_{\mathrm{V}} 1.2$ PY motif (PPSY ${ }_{1975}$ ), (James et al. 2015).

\section{Regulation of brain sodium channels by proteases}

Voltage-gated sodium channel density has long been known to be regulated by proteases under normal (Paillart et al. 1996) and stress conditions (Iwata et al. 2004). Among the most important proteases in mammalian cells stand the calpains, which target hundreds of proteins (Grimm et al. 2012). The group of Meany has revealed the bases of calpain-dependent proteolysis of $\mathrm{Na}_{\mathrm{V}} 1.2$.

Using rat brain homogenates, they showed that calpain cleaves $\mathrm{Na}_{\mathrm{V}} 1.2$ (but not $\mathrm{Na}_{\mathrm{V}} 1.1$ ) at two sites, i.e., the interdomain linkers between domains I and II, and between domains II and III (von Reyn et al. 2009). Intriguingly, most of the calpain sodium channel fragment products localise at the plasma membrane $6 \mathrm{~h}$ after calpain activation, and possibly interact (von Reyn et al. 2009). Perhaps the simplest explanation is that distinct sodium fragments still retain the protein-protein interactions that hold the sodium channel macromolecular complex together, and thus control the break-down of the complex. A more thought-provoking alternative is that sodium channel posttranslational proteolysis creates new proteins with modified biological activities.

The group of Meany has dissected the mechanisms of $\mathrm{Na}_{\mathrm{V}} 1.2$ proteolysis in cellular and mouse models of neuronal injury (von Reyn et al. 2012; Schoch et al. 2013), opening opportunities for treatment and therapy of traumatic brain injury. In this line, other researchers have recently described the beneficial effect of calpain inhibitors on brain sodium channel expression and electrophysiology in a model of diabetic neuropathy (Kharatmal et al. 2015).

The other example of sodium channel processing by proteases is the excision of the initiation methionine by aminopeptidases. This has been shown for $\mathrm{Na}_{\mathrm{V}} 1.5$ (followed by $N$-terminal acetylation of the resulting initiation alanine) in cardiac disease (Beltran-Alvarez et al. 2014).
Whether $\mathrm{Na}_{\mathrm{V}} 1.5$ or other $\mathrm{Na}_{\mathrm{V}}$ isoforms are devoid of Met residues (or post-translationally acetylated) in normal tissue is unknown.

\section{Conclusions and perspective}

Research in the voltage-gated sodium channel field has grown linearly for the last 20 years. While the interest in transcriptional mechanisms regulating sodium channel expression has also grown steadily, we have observed an exponential trend in the number of publications related to sodium channel post-translational regulation. We predict that this growth will keep pace over the coming years. The aim of this review was to provide the current state of the art of the transcriptional and post-translational regulation of sodium channels, and thus set the ground for further research opportunities and discoveries.

Our understanding of transcriptional mechanisms governing brain sodium channel expression is far from comprehensive, and the ongoing research efforts of the ENCODE Consortium will surely encourage groups around the globe to dissect the molecular mechanism controlling $\mathrm{Na}_{\mathrm{V}}$ transcription. Analogously, there are new questions in the field of PTM of sodium channels, in particular related to crosstalk among co-occurring types of PTM. As an example, the functional consequences of the interplay between phosphorylation and arginine methylation are intriguing, because the latter is thought to be a rather stable PTM (Bedford and Clarke 2009). The dynamic sequence of PTM events, thus, acquires vital relevance. Our incomplete understanding of proteolysis and degradation pathways of sodium channels also warrants further research in the area.

From the point of view of cell biology, biochemistry and electrophysiology, we predict that major advances in our understanding of $\mathrm{Na}_{\mathrm{V}}$ regulation will be made in two main directions. First, systems biology approaches will integrate knowledge on $\mathrm{Na}_{\mathrm{V}}$ biology, including transcriptional and post-translational regulation. This may be done using mathematical models and simulations of protein expression, function and degradation at the single molecule level, or, e.g., at the level of action potentials. Second, structural insights into whole sodium channel proteins, or isolated domains, will provide the framework to rationalise possible interactions between PTMs.

Additionally, research on $\mathrm{Na}_{\mathrm{V}}$ is intrinsically associated to biomedical sciences, given the prominent relevance of these channels in a range of neurological and cardiac disorders. In this respect, in the following years we expect reports on quantitative experiments identifying changes in PTM patterns in disease (some recent examples include Baek et al. 2014; and Herren et al. 2015). The effect of sodium channel proteolysis in major neurological diseases 
is also an emerging field of research (Corbett et al. 2013), which includes the identification of genetic mutations in proteases affecting sodium channel levels (Kim et al. 2014).

Acknowledgments DOO acknowledges Rivers State University of Science \& Technology Port Harcourt Nigeria, and TETfund Nigeria (Academic Staff Training and Development Unit) for funding. We are grateful to Sandra Jones and John Greenman (School of Biological, Biomedical and Environmental Sciences, University of Hull) for guidance.

\section{Compliance with ethical standards}

Conflict of interest The authors declare no conflict of interest. This review contains data published previously only. This article does not contain any studies with human participants or animals performed by any of the authors.

Open Access This article is distributed under the terms of the Creative Commons Attribution 4.0 International License (http://creativecommons.org/licenses/by/4.0/), which permits unrestricted use, distribution, and reproduction in any medium, provided you give appropriate credit to the original author(s) and the source, provide a link to the Creative Commons license, and indicate if changes were made.

\section{References}

Abriel H, Rougier JS, Jalife J (2015) Ion channel macromolecular complexes in cardiomyocytes: roles in sudden cardiac death. Circ Res 116(12):1971-1988

Ahern CA, Zhang JF, Wookalis MJ, Horn R (2005) Modulation of the cardiac sodium channel NaV1.5 by Fyn, a Src family tyrosine kinase. Circ Res 96(9):991-998

Baek JH, Cerda O, Trimmer JS (2011) Mass spectrometry-based phosphoproteomics reveals multisite phosphorylation on mammalian brain voltage-gated sodium and potassium channels. Semin Cell Dev Biol 22(2):153-159

Baek JH, Rubinstein M, Scheuer T, Trimmer JS (2014) Reciprocal changes in phosphorylation and methylation of mammalian brain sodium channels in response to seizures. J Biol Chem 289(22):15363-15373

Baroni D, Picco C, Barbieri R, Moran O (2014) Antisense-mediated post-transcriptional silencing of SCN1B gene modulates sodium channel functional expression. Biol Cell 106(1):13-29

Baycin-Hizal D, Gottschalk A, Jacobson E, Mai S, Wolozny D, Zhang H, Krag SS, Betenbaugh MJ (2014) Physiologic and pathophysiologic consequences of altered sialylation and glycosylation on ion channel function. Biochem Biophys Res 453(2):243-253

Beacham D, Ahn M, Catterall WA, Scheuer T (2007) Sites and molecular mechanisms of modulation of $\mathrm{Na}(\mathrm{v}) 1.2$ channels by Fyn tyrosine kinase. J Neurosci 27(43):11543-11551

Bedford MT, Clarke SG (2009) Protein arginine methylation in mammals: who, what, and why. Mol Cell 33(1):1-13

Beltran-Alvarez P, Pagans S, Brugada R (2011) The cardiac sodium channel is post-translationally modified by arginine methylation. J Proteome Res 10(8):3712-3719

Beltran-Alvarez P, Espejo A, Schmauder R, Beltran C, Mrowka R, Linke T, Batlle M, Pérez-Villa F, Pérez GJ, Scornik FS, Benndorf K, Pagans S, Zimmer T, Brugada R (2013) Protein arginine methyl transferases-3 and -5 increase cell surface expression of cardiac sodium channel. FEBS Lett 587(19):3159-3165
Beltran-Alvarez P, Tarradas A, Chiva C, Pérez-Serra A, Batlle M, Pérez-Villa F, Schulte U, Sabidó E, Brugada R, Pagans S (2014) Identification of $N$-terminal protein acetylation and arginine methylation of the voltage-gated sodium channel in end-stage heart failure human heart. J Mol Cell Cardiol 76:126-129

Beltran-Alvarez P, Feixas F, Osuna S, Díaz-Hernández R, Brugada R, Pagans S (2015) Interplay between R513 methylation and S516 phosphorylation of the cardiac voltage-gated sodium channel. Amino Acids 47(2):429-434

Berendt FJ, Park KS, Trimmer JS (2010) Multisite phosphorylation of voltage-gated sodium channel alpha subunits from rat brain. J Proteome Res 9(4):1976-1984

Bosmans F, Milescu M, Swartz KJ (2011) Palmitoylation influences the function and pharmacology of sodium channels. Proc Natl Acad Sci USA 108(50):20213-20218

Buchner DA, Trudeau M, Meisler MH (2003) SCNM1, a putative RNA splicing factor that modifies disease severity in mice. Science 301(5635):967-969

Cantrell AR, Catterall WA (2001) Neuromodulation of $\mathrm{Na}+$ channels: an unexpected form of cellular plasticity. Nat Rev Neurosci 2(6):397-407

Catterall WA (2015) Finding channels. J Biol Chem. doi:10.1074/jbc. X115.683383

Cerda O, Baek JH, Trimmer JS (2011) Mining recent brain proteomic databases for ion channel phosphosite nuggets. J Gen Physiol 137(1):3-16

Chen C, Westenbroek RE, Xu X, Edwards CA, Sorenson DR, Chen Y, McEwen DP, O'Malley HA, Bharucha V, Meadows LS, Knudsen GA, Vilaythong A, Noebels JL, Saunders TL, Scheuer T, Shrager P, Catterall WA, Isom LL (2004) Mice lacking sodium channel beta1 subunits display defects in neuronal excitability, sodium channel expression, and nodal architecture. J Neurosci 24(16):4030-4042

Chen Y, Yu FH, Sharp EM, Beacham D, Scheuer T, Catterall WA (2008) Functional properties and differential neuromodulation of $\mathrm{Na}(\mathrm{v}) 1.6$ channels. Mol Cell Neurosci 38(4):607-615

Copley RR (2004) Evolutionary convergence of alternative splicing in ion channels. Trends Genet 20(4):171-176

Corbett BF, Leiser SC, Ling HP, Nagy R, Breysse N, Zhang X, Hazra A, Brown JT, Randall AD, Wood A, Pangalos MN, Reinhart PH, Chin J (2013) Sodium channel cleavage is associated with aberrant neuronal activity and cognitive deficits in a mouse model of Alzheimer's disease. J Neurosci 33(16):7020-7026

Cui ZJ, Han ZQ, Li ZY (2012) Modulating protein activity and cellular function by methionine residue oxidation. Amino Acids 43(2):505-517

Dib-Hajj SD, Yang Y, Black JA, Waxman SG (2013) The Na(V)1.7 sodium channel: from molecule to man. Nat Rev Neurosci 14(1):49-62

Dong ZF, Tang LJ, Deng GF, Zeng T, Liu SJ, Wan RP, Liu T, Zhao QH, Yi YH, Liao WP, Long YS (2014) Transcription of the human sodium channel SCN1A gene is repressed by a scaffolding protein RACK1. Mol Neurobiol 50(2):438-448

Drews VL, Lieberman AP, Meisler MH (2005) Multiple transcripts of sodium channel SCN8A $(\mathrm{Na}(\mathrm{V}) 1.6)$ with alternative $5^{\prime}$ - and $3^{\prime}$-untranslated regions and initial characterization of the SCN8A promoter. Genomics 85(2):245-257

Drews VL, Shi K, de Haan G, Meisler MH (2007) Identification of evolutionarily conserved, functional noncoding elements in the promoter region of the sodium channel gene SCN8A. Mamm Genome 18(10):723-731

Dustrude ET, Wilson SM, Ju W, Xiao Y, Khanna R (2013) CRMP2 protein SUMOylation modulates NaV1.7 channel trafficking. J Biol Chem 288(34):24316-24331

Few WP, Scheuer T, Catterall WA (2007) Dopamine modulation of neuronal $\mathrm{Na}(+)$ channels requires binding of A kinase-anchoring 
protein 15 and PKA by a modified leucine zipper motif. Proc Natl Acad Sci USA 104(12):5187-5192

Fotia AB, Ekberg J, Adams DJ, Cook DI, Poronnik P, Kumar S (2004) Regulation of neuronal voltage-gated sodium channels by the ubiquitin-protein ligases Nedd4 and Nedd4-2. J Biol Chem 279(28):28930-28935

Gasser A, Cheng X, Gilmore ES, Tyrrell L, Waxman SG, DibHajj SD (2010) Two Nedd4-binding motifs underlie modulation of sodium channel Nav1.6 by p38 MAPK. J Biol Chem 285(34):26149-26161

Gehman LT, Meera P, Stoilov P, Shiue L, O'Brien JE, Meisler MH, Ares M Jr, Otis TS, Black DL (2012) The splicing regulator Rbfox 2 is required for both cerebellar development and mature motor function. Genes Dev 26(5):445-460

Goel P, Manning JA, Kumar S (2015) NEDD4-2 (NEDD4L): the ubiquitin ligase for multiple membrane proteins. Gene 557(1):1-10

Grimm S, Höhn A, Grune T (2012) Oxidative protein damage and the proteasome. Amino Acids 42(1):23-38

Guo A, Gu H, Zhou J, Mulhern D, Wang Y, Lee KA, Yang V, Aguiar M, Kornhauser J, Jia X, Ren J, Beausoleil SA, Silva JC, Vemulapalli V, Bedford MT, Comb MJ (2014) Immunoaffinity enrichment and mass spectrometry analysis of protein methylation. Mol Cell Proteomics 13(1):372-387

Gustafson TA, Clevinger EC, O’Neill TJ, Yarowsky PJ, Krueger BK (1993) Mutually exclusive exon splicing of type III brain sodium channel alpha subunit RNA generates developmentally regulated isoforms in rat brain. J Biol Chem 268(25):18648-18653

Hallaq H, Yang Z, Viswanathan PC, Fukuda K, Shen W, Wang DW, Wells KS, Zhou J, Yi J, Murray KT (2006) Quantitation of protein kinase A-mediated trafficking of cardiac sodium channels in living cells. Cardiovasc Res 72(2):250-261

Heinzen EL, Yoon W, Tate SK, Sen A, Wood NW, Sisodiya SM, Goldstein DB (2007) Nova2 interacts with a cis-acting polymorphism to influence the proportions of drug-responsive splice variants of SCN1A. Am J Hum Genet 80(5):876-883

Herren AW, Weber DM, Rigor RR, Margulies KB, Phinney BS, Bers DM (2015) CaMKII phosphorylation of $\mathrm{Na}(\mathrm{V}) 1.5$ : novel in vitro sites identified by mass spectrometry and reduced S516 phosphorylation in human heart failure. J Proteome Res 14(5):2298-2311

Hien YE, Montersino A, Castets F, Leterrier C, Filhol O, Vacher H, Dargent B (2014) CK2 accumulation at the axon initial segment depends on sodium channel Nav1. FEBS Lett 588(18):3403-3408

Hornbeck PV, Zhang B, Murray B, Kornhauser JM, Latham V, Skrzypek E (2015) PhosphoSitePlus, 2014: mutations, PTMs and recalibrations. Nucleic Acids Res 43(1):D512-D520

Iqbal SM, Andavan GS, Lemmens-Gruber R (2015) Differential modulation of fast inactivation in cardiac sodium channel splice variants by fyn tyrosine kinase. Cell Physiol Biochem 37(3):825-837

Iwata A, Stys PK, Wolf JA, Chen XH, Taylor AG, Meaney DF, Smith DH (2004) Traumatic axonal injury induces proteolytic cleavage of the voltage-gated sodium channels modulated by tetrodotoxin and protease inhibitors. J Neurosci 24(19):4605-4613

James TF, Nenov MN, Wildburger NC, Lichti CF, Luisi J, Vergara F, Panova-Electronova NI, Nilsson CL, Rudra JS, Green TA, Labate D, Laezza F (2015) The Nav1.2 channel is regulated by GSK3. Biochim Biophys Acta 1850(4):832-844

Kasteleijn-Nolst Trenité DG, Volkers L, Strengman E, Schippers HM, Perquin W, de Haan GJ, Gkountidi AO, van't Slot R, de Graaf SF, Jocic-Jakubi B, Capovilla G, Covanis A, Parisi P, Veggiotti P, Brinciotti M, Incorpora G, Piccioli M, Cantonetti L, Berkovic SF, Scheffer IE, Brilstra EH, Sonsma AC, Bader AJ, de Kovel CG, Koeleman BP (2015) Clinical and genetic analysis of a family with two rare reflex epilepsies. Seizure 29:90-96

Kharatmal SB, Singh JN, Sharma SS (2015) Calpain inhibitor, MDL 28170 confer electrophysiological, nociceptive and biochemical improvement in diabetic neuropathy. Neuropharmacology 97:113-121

Khoury GA, Baliban RC, Floudas CA. (2011) Proteome-wide posttranslational modification statistics: frequency analysis and curation of the swiss-prot database. Sci Rep, 1 pii: srep00090

Kim DY, Ingano LA, Carey BW, Pettingell WH, Kovacs DM (2005) Presenilin/gamma-secretase-mediated cleavage of the voltagegated sodium channel beta2-subunit regulates cell adhesion and migration. J Biol Chem 280(24):23251-23261

Kim DY, Carey BW, Wang H, Ingano LA, Binshtok AM, Wertz MH, Pettingell WH, He P, Lee VM, Woolf CJ, Kovacs DM (2007) BACE1 regulates voltage-gated sodium channels and neuronal activity. Nat Cell Biol 9(7):755-764

Kim DY, Gersbacher MT, Inquimbert P, Kovacs DM (2011) Reduced sodium channel $\mathrm{Na}(\mathrm{v}) 1.1$ levels in BACE1-null mice. J Biol Chem 286(10):8106-8116

Kim DY, Wertz MH, Gautam V, D’Avanzo C, Bhattacharyya R, Kovacs DM (2014) The E280A presenilin mutation reduces voltage-gated sodium channel levels in neuronal cells. Neurodegener Dis 13(2-3):64-68

Laedermann CJ, Syam N, Pertin M, Decosterd I, Abriel H (2013) $\beta 1$ and $\beta 3$-voltage-gated sodium channel subunits modulate cell surface expression and glycosylation of Nav1.7 in HEK293 cells. Front Cell Neurosci 7:137

Le Gal F, Salzmann A, Crespel A, Malafosse A (2011) Replication of association between a SCN1A splice variant and febrile seizures. Epilepsia 52(10):e135-e138

Li M, West JW, Lai Y, Scheuer T, Catterall WA (1992) Functional modulation of brain sodium channels by cAMP-dependent phosphorylation. Neuron 8(6):1151-1159

Li M, West JW, Numann R, Murphy BJ, Scheuer T, Catterall WA (1993) Convergent regulation of sodium channels by protein kinase $\mathrm{C}$ and cAMP-dependent protein kinase. Science 261(5127):1439-1442

Li HJ, Wan RP, Tang LJ, Liu SJ, Zhao QH, Gao MM, Yi YH, Liao WP, Sun XF, Long YS (2015) Alteration of Scn3a expression is mediated via $\mathrm{CpG}$ methylation and $\mathrm{MBD} 2$ in mouse hippocampus during postnatal development and seizure condition. Biochim Biophys Acta 1849(1):1-9

Long YS, Zhao QH, Su T, Cai YL, Zeng Y, Shi YW, Yi YH, Chang $\mathrm{HH}$, Liao WP (2008) Identification of the promoter region and the $5^{\prime}$-untranslated exons of the human voltage-gated sodium channel Nav1.1 gene (SCN1A) and enhancement of gene expression by the $5^{\prime}$-untranslated exons. J Neurosci Res 86(15):3375-3381

Lossin C (2009) A catalog of SCN1A variants. Brain Dev 31(2):114-130

Lu CM, Eichelberger JS, Beckman ML, Schade SD, Brown GB (1998) Isolation of the $5^{\prime}$-flanking region for human brain sodium channel subtype II alpha-subunit. J Mol Neurosci 11(3):179-182

Marionneau C, Abriel H (2015) Regulation of the cardiac Na+ channel NaV1.5 by post-translational modifications. J Mol Cell Cardiol 82:36-47

Marionneau C, Lichti CF, Lindenbaum P, Charpentier F, Nerbonne JM, Townsend RR, Mérot J (2012) Mass spectrometry-based identification of native cardiac Nav1.5 channel $\alpha$ subunit phosphorylation sites. J Proteome Res 11(12):5994-6007

Martin MS, Tang B, Ta N, Escayg A (2007) Characterization of $5^{\prime}$ untranslated regions of the voltage-gated sodium channels SCN1A, SCN2A, and SCN3A and identification of cis-conserved noncoding sequences. Genomics 90(2):225-235

Mercier A, Clément R, Harnois T, Bourmeyster N, Bois P, Chatelier A (2015) Nav1.5 channels can reach the plasma membrane through distinct $N$-glycosylation states. Biochim Biophys Acta 1850(6):1215-1223 
Namadurai S, Yereddi NR, Cusdin FS, Huang CL, Chirgadze DY, Jackson AP (2015) A new look at sodium channel $\beta$ subunits. Open Biol 5(1):140192

Numann R, Catterall WA, Scheuer T (1991) Functional modulation of brain sodium channels by protein kinase $\mathrm{C}$ phosphorylation. Science 254(5028): 115-118

Ogino K, Low SE, Yamada K, Saint-Amant L, Zhou W, Muto A, Asakawa K, Nakai J, Kawakami K, Kuwada JY, Hirata H (2015) RING finger protein 121 facilitates the degradation and membrane localization of voltage-gated sodium channels. Proc Natl Acad Sci USA 112(9):2859-2864

Paillart C, Boudier JL, Boudier JA, Rochat H, Couraud F, Dargent B (1996) Activity-induced internalization and rapid degradation of sodium channels in cultured fetal neurons. J Cell Biol 134(2):499-509

Renganathan M, Cummins TR, Waxman SG (2002) Nitric oxide blocks fast, slow, and persistent $\mathrm{Na}+$ channels in C-type DRG neurons by $S$-nitrosylation. J Neurophysiol 87(2):761-775

Rogers LD, Overall CM (2013) Proteolytic post-translational modification of proteins: proteomic tools and methodology. Mol Cell Proteomics 12(12):3532-3542

Rougier JS, van Bemmelen MX, Bruce MC, Jespersen T, Gavillet B, Apothéloz F, Cordonier S, Staub O, Rotin D, Abriel H (2005) Molecular determinants of voltage-gated sodium channel regulation by the Nedd4/Nedd4-like proteins. Am J Physiol Cell Physiol 288(3):C692-C701

Sarao R, Gupta SK, Auld VJ, Dunn RJ (1991) Developmentally regulated alternative RNA splicing of rat brain sodium channel mRNAs. Nucleic Acids Res 19(20):5673-5679

Schade SD, Brown GB (2000) Identifying the promoter region of the human brain sodium channel subtype II gene (SCN2A). Brain Res Mol Brain Res 81(1-2):187-190

Schlachter K, Gruber-Sedlmayr U, Stogmann E, Lausecker M, Hotzy C, Balzar J, Schuh E, Baumgartner C, Mueller JC, Illig T, Wichmann HE, Lichtner P, Meitinger T, Strom TM, Zimprich A, Zimprich F (2009) A splice site variant in the sodium channel gene SCN1A confers risk of febrile seizures. Neurology. 72(11):974-978

Schmidt JW, Catterall WA (1987) Palmitylation, sulfation, and glycosylation of the alpha subunit of the sodium channel. Role of posttranslational modifications in channel assembly. J Biol Chem 262(28):13713-13723

Schoch KM, von Reyn CR, Bian J, Telling GC, Meaney DF, Saatman KE (2013) Brain injury-induced proteolysis is reduced in a novel calpastatin-overexpressing transgenic mouse. J Neurochem 125(6):909-920
Tate SK, Depondt C, Sisodiya SM, Cavalleri GL, Schorge S, Soranzo N, Thom M, Sen A, Shorvon SD, Sander JW, Wood NW, Goldstein DB (2005) Genetic predictors of the maximum doses patients receive during clinical use of the anti-epileptic drugs carbamazepine and phenytoin. Proc Natl Acad Sci USA 102:5507-5512

Thompson CH, Kahlig KM, George AL Jr (2011) SCN1A splice variants exhibit divergent sensitivity to commonly used antiepileptic drugs. Epilepsia 52(5):1000-1009

van Bemmelen MX, Rougier JS, Gavillet B, Apothéloz F, Daidié D, Tateyama M, Rivolta I, Thomas MA, Kass RS, Staub O, Abriel H (2004) Cardiac voltage-gated sodium channel Nav1.5 is regulated by Nedd4-2 mediated ubiquitination. Circ Res 95(3):284-291

von Reyn CR, Spaethling JM, Mesfin MN, Ma M, Neumar RW, Smith DH, Siman R, Meaney DF (2009) Calpain mediates proteolysis of the voltage-gated sodium channel alpha-subunit. J Neurosci 29(33): 10350-10356

von Reyn CR, Mott RE, Siman R, Smith DH, Meaney DF (2012) Mechanisms of calpain mediated proteolysis of voltage gated sodium channel $\alpha$-subunits following in vitro dynamic stretch injury. J Neurochem 121(5):793-805

West JW, Numann R, Murphy BJ, Scheuer T, Catterall WA (1991) A phosphorylation site in the $\mathrm{Na}+$ channel required for modulation by protein kinase C. Science 254(5033):866-868

Wittmack EK, Rush AM, Hudmon A, Waxman SG, Dib-Hajj SD (2005) Voltage-gated sodium channel Nav1.6 is modulated by p38 mitogen-activated protein kinase. J Neurosci 25(28):6621-6630

Wong HK, Sakurai T, Oyama F, Kaneko K, Wada K, Miyazaki H, Kurosawa M, De Strooper B, Saftig P, Nukina N (2005) beta Subunits of voltage-gated sodium channels are novel substrates of beta-site amyloid precursor protein-cleaving enzyme (BACE1) and gamma-secretase. J Biol Chem 280(24):23009-23017

Yu FH, Catterall WA (2003) Overview of the voltage-gated sodium channel family. Genome Biol 4(3):207

Zhou J, Shin HG, Yi J, Shen W, Williams CP, Murray KT (2002) Phosphorylation and putative ER retention signals are required for protein kinase A-mediated potentiation of cardiac sodium current. Circ Res 91(6):540-546

Zilberter YuI, Starmer CF, Starobin J, Grant AO (1994) Late Na channels in cardiac cells: the physiological role of background $\mathrm{Na}$ channels. Biophys J 67(1):153-160 